\title{
On the Four-Valuedness of Twistors
}

\author{
C. J. S. Clarke \\ Department of Mathematics, University of York, Heslington, York Y01 5DD, England
}

\begin{abstract}
The spinors on compactified Minkowski space, in terms of which twistor theory is formulated, are really $U$-spinors. In this light zero-mass fields have no Grgin discontinuity.
\end{abstract}

I shall examine the spinors which are induced on compactified Minkowsky space, $M^{c}$, by twistors. The notation will follow [3], to which the reader is referred for the basic facts of twistor theory. Note in particular that I shall mainly be using concrete indices ${ }^{1}$, since the abstract index notation of [4] presupposes the existence of some particular spin structure; and it is precisely this that I wish to explore.

If $Z$ and $W$ are two twistors with components $\left(Z^{\alpha}\right)=\left(\eta^{\mathfrak{A}}, l_{\mathfrak{X}^{\prime}}\right),\left(W^{\alpha}\right)=\left(\xi^{\mathfrak{N}}, \sigma_{\mathfrak{X}^{\prime}}\right)$, then they determine the point $x(Z, W)$ in Minkowski space $M$ whose components are

$$
x^{\mathfrak{a}}=-i \sigma^{\mathfrak{a} \mathfrak{U} \mathfrak{X}^{\prime}}\left(\eta_{\mathfrak{W}} \sigma_{\mathfrak{X}^{\prime}}-\xi_{\mathfrak{Q}} l_{\mathfrak{X}^{\prime}}\right) / l_{\mathfrak{Y}^{\prime}} \sigma^{\mathfrak{Y}^{\prime}}
$$

provided that $l_{\mathfrak{Y}^{\prime}} \sigma^{\mathfrak{Y}^{\prime}} \neq 0$. Then an element $g$ of the twistor transformation group $\mathrm{SU}(2,2)[5]$ determines a local conformal transformation $\tilde{g}$ on $M$ by

$$
\tilde{g}(x(Z, W))=x(g(Z), g(W)),
$$

in a domain where both sides are defined.

The two pairs of numbers which make up the components of a twistor are interpreted on $M$ as the components of spinors with respect to a fixed coordinate basis. Not only are they related to vectors by (1), but for any Poincare transformation $\tilde{g}$ on $M$ one can find a $g$ which acts on these twistor components in the way appropriate to the spinor interpretation. Moreover, this action extends to conformal transformations, under which the $l_{\mathfrak{X}^{\prime}}$ and $\eta^{\mathscr{I}}$ transform as the components of spinors on $M$ of conformal weight 1 (i.e. under dilatation by a factor $\theta$ they acquire a factor $\theta^{-1}$ ). Hence they are describable in terms of the conformal metric alone, and so can be defined on the image of $M$ in $M^{c}$. However, it is well known ([3],

\footnotetext{
For typographical reasons concrete twistor indices are represented by $\alpha, \beta$ etc., instead of the Hebrew of [3].
} 
p. 258) that the extensions of these spinors to $M^{c}$, with the extension of the $\tilde{g}$ to globally defined transformations, leads to a four-valuedness.

Consider [5] the one-parameter family of transformations $g(\theta) \in \mathrm{SU}(2,2)$ given by

$$
\left[g(\theta)_{\beta}^{\alpha}\right]=\left[\begin{array}{cccc}
e^{-i \theta} \cos 2 \theta & 0 & -i e^{-i \theta} \sin 2 \theta & 0 \\
0 & e^{i \theta} & 0 & 0 \\
-i e^{-i \theta} \sin 2 \theta & 0 & e^{-i \theta} \cos 2 \theta & 0 \\
0 & 0 & 0 & e^{i \theta}
\end{array}\right]
$$

Then $g(\pi / 2)=i \times$ identity, $\tilde{g}(\pi / 2)=$ identity and the action of $\tilde{g}(\theta)$ carries the origin of coordinates in $M$ down to $\mathscr{I}^{-} \equiv \mathscr{I}^{+}$and back down to the origin. If we choose a pseudo-orthonormal frame at the origin it will be dragged round this path by $\tilde{g}(\theta)$, giving for each $\theta$ a conformally pseudoorthonormal frame $e_{\theta}$ on $M^{c}$.

On any reasonable interpretation, we should expect $g(\theta)$ to transform spinors on $M^{c}$ continuously, so that, considering, say, the last two twistor components, $\lim _{\theta>\pi / 2} g(\theta)_{\mathfrak{Q}^{\prime}}{ }^{\mathfrak{B}^{\prime}} \eta_{\mathfrak{B}^{\prime}}$ and $\lim _{\theta \succ \pi / 2} g(\theta)_{\mathfrak{F}^{\prime}}{ }^{\mathfrak{B}^{\prime}} \eta_{\mathfrak{B}^{\prime}}$ represent the same spinor. But we cannot compare their components in the coordinate basis of $M$ because this leads to a spinor basis in $M^{c}$ which is discontinuous on $\mathscr{I}$; instead we transform to the basis $e_{\theta}$.

A calculation shows that the components of the $g(\theta)$-dragged spinor $g(\theta)_{\mathfrak{A}^{\prime}}{ }^{\mathfrak{B}^{\prime}} \eta_{\mathfrak{B}^{\prime}}$ in the $g(\theta)$-frame are constant as $\theta$ varies from 0 to $\pi / 4$, or from $\pi / 4$ to $\pi / 2$. Thus on return to the origin at $\theta=\pi / 2$, the $e_{\theta}$ components are unchanged, while the coordinate-basis components (i.e. the $e_{0}$-components) have become multiplied by $+i$. If we are to extend spinors to $M^{c}$, the frame $e_{\pi / 2}$ is not, as far as spinors are concerned, the same frame as $e_{0}$. Just as a frame acquires a spin-entanglement [6] on rotation through $2 \pi$, so $e_{\theta}$ on passing from $e_{0}$ to $e_{\pi / 2}$ acquires a half-entanglement - let us call it a spin-rotation of $\pi$ (as pointed out in [3], loc. cit.). While not allowed for spinors in the usual sense, this is permissible for $U$-spinors.

We recall [1] that $U$-spinors are defined on a space-time $X$ by extending the bundle $L(X)$ of all pseudo-orthonormal frames to a $U \operatorname{Spin}_{+}(1,3)$-bundle $U(X)$, where $U \operatorname{Spin}_{+}(1,3) \simeq\left(\operatorname{Spin}_{+}(1,3) \times U(1)\right) / Z_{2}$ (with the non-trivial factorisation); just as spinors are defined by extending $L(X)$ to a $\operatorname{Spin}_{+}(1,3)$-bundle $S(X)$, Spin $_{+}(1,3) \simeq S L(2, C)$ being the covering group of the Lorentz group. If a loop in $L(X)$ is lifted to $U(X)$ it defines a transformation in $U(1)$ which, in the case of spinors on $M^{c}$ belongs to the subgroup $G=\{1, i,-1,-i\}$. In this case the group of $U(X)$ reduces to $\left(\operatorname{Spin}_{+}(1,3) \times G\right) / Z_{2}$ and we have generalised spinors, as described in [2]. [Note that any generalised spinor bundle $E$ with group

$\left(\operatorname{Spin}_{+}(1,3) \times H\right) / Z_{2}$

can be extended to a $U$ Spin-bundle by forming $(E \times U(1)) / H$. On the other hand, the two-plane bundles over $S^{2}$ with no spin structure [7] do not admit a generalised spin structure but do admit $U$-spinors, since their dimension-three cohomology is obviously trivial. Thus $U$-spinors are more general than generalised spinors.]

Finally, consider zero-mass fields on $M^{c}$. These are specified by

$$
\phi_{A B \ldots D}\left(x^{a}\right)=\oint W_{A} W_{B} \ldots W_{D} f\left(W_{\alpha}\right) I^{\beta \gamma} W_{\beta} d W_{\gamma}
$$


where $W_{\alpha}$ is a lower-index twistor (an element of the space dual to upper-index twistors), restricted by $\left(W_{\alpha}\right)=\left(W_{\mathfrak{Q}}, i x^{\mathfrak{B B} \mathfrak{B}^{\prime}} W_{\mathfrak{B}}\right)$. As usual, $f$ is of homogeneity degree $-n-2$, where $n$ is the valence of $\phi$. As before, transform to the $e_{\theta}$ basis by the conformal spinor transformation $S_{0 \mathfrak{B}}{ }^{\mathfrak{A}}$. Since $S_{\theta \mathfrak{B}}{ }^{\mathfrak{A}} \frac{{ }^{\theta \mathfrak{A}}}{\mathfrak{c}}=$ identity (the bar denoting complex conjugation, needed for passing from $l_{\mathfrak{x}^{\prime}}$ to $W_{\mathfrak{Q}}$ ), on applying $g(\theta)$ to $W$ we have the $e_{\theta}$-components given by

$$
\left.\tilde{\phi}_{\mathfrak{Q} \mathfrak{B} \ldots \mathcal{D}}^{\theta}\left(\tilde{g}(\theta)\left(x^{a}\right)\right)=\oint W_{\mathfrak{Q}} W_{\mathfrak{B}} \ldots W_{\mathfrak{D}} f\left(\overline{g(\theta)} W_{\alpha}\right) \times I^{\beta \alpha} \overline{g(\theta)}\right)_{\alpha} \overline{g(\theta)}_{\beta}{ }^{\delta} W_{\delta} d W_{\gamma}
$$

the contour being homologous to that in (2). Here the tilde and superscript $\theta$ on $\phi$ simply indicate that the components are expressed in the $e_{\theta}$ basis.

The discontinuity across $\mathscr{I}$ is obtained by comparing values at $\theta=-\pi / 4+0$ and $\theta=\pi / 4-0\left(\mathscr{I}^{+}\right.$and $\mathscr{I}^{-}$, respectively). Recalling that $g(\pi / 2)=i g(0)$, and hence $g(\pi / 4)=i g(-\pi / 4)$, and using the homogeneity of $f$, we find

$$
\tilde{\phi}_{\mathfrak{Q B B}}^{\pi / 4} \ldots \mathfrak{D}\left(y^{a}\right)=i^{n} \tilde{\phi}_{\mathfrak{Q B B}}^{-\pi / 4} \ldots \mathfrak{D}\left(y^{a}\right)
$$

where $y^{a}=\lim _{\theta \rightarrow \pi / 4} g(\theta) x^{a} \in M^{c}$. But we already have, from considering the spinrotation of $e_{\theta}$, that

$$
\tilde{\eta}_{\mathfrak{X}^{\prime}}^{\pi / 2}(0)=-i \tilde{\eta}_{\mathfrak{X}^{\prime}}^{0}(0) \text {. }
$$

Thus the spin-rotation expressed in (3) is precisely that which is expected for a continuous spinor field of the indicated type, found by conjugating (4) and taking an $n$-fold tensor product.

In short, there is no Grgin discontinuity.

\section{References}

1. Whiston, G.S.: Lorentzian Characteristic Classes; to appear in General Relativity and Gravitation

2. Clarke, C.J.S.: General Relativity and Gravitation 5, 163-167 (1974)

3. Penrose,R., MacCallum,M.A.H.: Twistor theory: an approach to the quantisation of fields and space-time. Phys. Rep. 6, 241-316 (1972)

4. Penrose, R.: Structure of Space-Time. In: Battelle Rencontres, p. 121-241. New York: Benjamin 1968

5. Penrose, R.: J. Math. Phys. 8, 345-366 (1967)

6. Wheeler, J.A.: Superspace and Quantum Geometrodynamics. In: Battelle Rencontres, p. $242-307$. New York: Benjamin 1968

7. Geroch, R.: J. Math. Phys. 9, 1739-1744 (1970)

Communicated by J. Ehlers

Received July 19, 1975 
